\title{
Discovery and technology for human health
}

\author{
Nature Biomedical Engineering will bring the convergence of fields in the health and physical sciences into \\ the spotlight.
}

Breakthrough discovery and invention often emerge at the interface between disciplines. As scientific research becomes ever more collaborative (R. van Noorden, Nature 525, 306-307; 2015), scientific and technological developments rooted in interdisciplinarity are poised to increasingly fuel human endeavour. Biomedical engineering - an inherently interdisciplinary subject area that aims to improve human health - indeed thrives on the convergence of research fields.

For example, biomedical engineers working on cellular self-assembly and bioprinting take advantage of knowledge that cuts across tissue engineering, materials science and regenerative medicine. Those devising better approaches to deliver immunotherapeutic drugs need to integrate concepts from cancer immunotherapy and nanotechnology. Researchers aiming to help surgeons make informed decisions faster and more accurately are drawing on techniques from artificial intelligence and on the knowledge of the pathologist. For creative minds with problem-solving skills - a typical engineering mind-set methods and approaches from the physical, biological and medical sciences can offer a fruitful playground at junctures between neuroscience and machine/brain interfaces, nanotechnology and biotechnology, and therapy and diagnostics.

Biomedical engineering started off with a clinical focus. Artificial joints, magnetic resonance imaging, heart pacemakers, heart-lung machines and angioplasties have prolonged the lives of patients, or improved their quality of life. These major advances of biomedical engineering rely on findings or developments that stem from basic research in the physical sciences and in human biology or medicine: for example, a total hip replacement requires biocompatible ceramic materials with low wear rates; efficient magnetic resonance imaging uses metal nanoparticles with high relaxivity as contrast agents; and pacemaker electrodes have to be made of materials that inhibit fibrosis. Heart-lung machines are designed to prevent clot formation, haemolysis and metabolic acidosis, and catheters for peripheral or coronary angioplasties may soon include electrodes for measuring electrophysiological data.

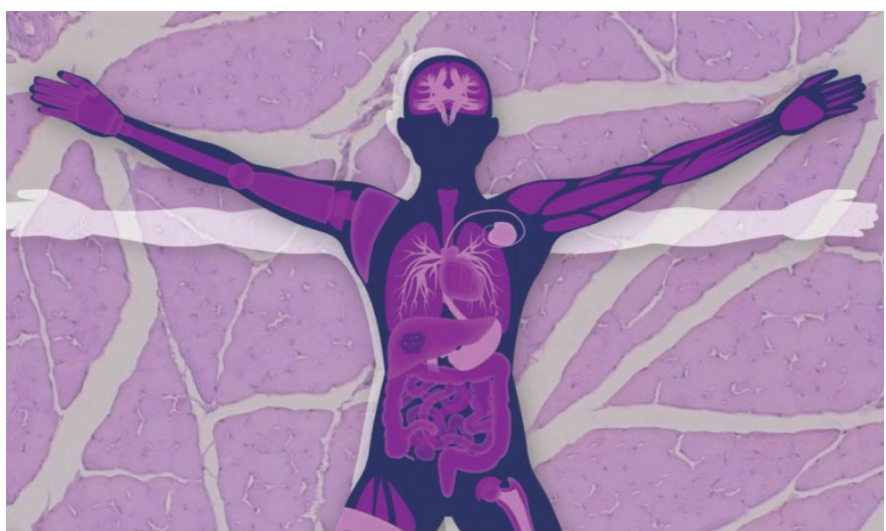

inspiration from ancient whirligigs, Manu Prakash and colleagues designed a hand-held spinning disk out of paper and string that can rival the performance of a standard (yet vastly more expensive) centrifuge (Article 0009). And fluorescent nanoparticles designed by Jinming Gao and colleagues

Hence, clinical developments often inspire further improvements in methodology and generate hypotheses to be tested in the laboratory. Conversely, fundamental advances in biology, medicine, materials science, chemistry and biophysics can lead to the development of technology that may eventually reach the clinic. Such a feedback loop has driven the advance of biomaterials and tissue engineering since the late 1980s, and has prompted biomedical engineers to piggyback on the recent growth of 'omics' fields, systems biology and synthetic biology to develop diagnostic and therapeutic tools. Yet reaching the clinic is certainly not the only goal. Biomedical engineers will move the needle in global health, by devising hardware, software and methods with the appropriate trade-offs in design and functionality necessary for the field and the home, especially in poorresource settings.

This inaugural issue of Nature Biomedical Engineering pays homage to the interdisciplinarity of the subject area and the ingenuity of biomedical engineers. The issue unveils fundamental advances in the fate of implanted biomaterials and in the generation of cardiac stem cells, methodological approaches for assessing tumour mechanopathology, and technical developments in image-guided surgery and in diagnostics for global health. It also highlights a range of diseases and diseaserelated topics - diabetes, cancer, infectious diseases, cardiology and immunology — as well as regulatory aspects of cardiovascular devices and neurocognitive considerations of body-augmentation technology. By taking to mimic the functionality of a transistor so as to light up tumours only in the presence of acidic extracellular $\mathrm{pH}$ will help surgeons find tumour nodules and guide tumour resection (Article 0006).

The need to visualize molecules, cells and tissues puts imaging techniques centre stage in biomedical engineering research. To highlight the significance of imaging, in this first issue we have included two Review Articles - one on imaging methods in diagnostics, therapy and surgery, and a second on near-infrared imaging - that overview a range of imaging modalities. We have also curated a collection of Articles, Review Articles and News \& Views published in Nature journals in the past two years in the field of optical imaging for diagnostics (http://www.nature.com/diagnostic-imaging).

By publishing content that traverses field boundaries, at Nature Biomedical Engineering we aim to help build bridges between bench researchers, clinicians and medical engineers. This online-only journal has been designed to also attract the most significant developments in the full spectrum of research in biomedical engineering, and we will strive to inspire biomedical engineers to help solve outstanding health challenges by disseminating the successes of the discipline to the wider scientific community. To this end, we have also launched a free-to-access, collaborative online site (https://nbmecommunity.nature.com) for our readers and authors to engage in enriching discussions and to contribute with research news and stories. We look forward to welcoming you. 